\title{
Low Frequency Broadband InGaAs/InAIAs Low Noise Amplifiers for SKA
}

\author{
S. Boulay ${ }^{\star}$, B. Boudjelida, S. Arshad, N. Ahmad, M. Missous
}

School of Electrical and Electronic Engineering, University of Manchester, UK.

\begin{abstract}
A new high breakdown InP pHEMT has been used in the design, fabrication and testing of wideband Low noise amplifiers for use in the aperture array concept of the SKA. This optically defined $1 \mu \mathrm{m}$ gate, $800 \mu \mathrm{m}$ gate width process has permitted the realisation of low noise transistors with NFmin $<0.4 \mathrm{~dB}(28 \mathrm{~K})$ at $2 \mathrm{GHz}$ and lower NFmin values at lower frequencies. The large gate width has been instrumental in realising low noise resistances.

We report here on DC, RF and noise performances of novel MMIC Low Noise Amplifiers based on InGaAs/InAlAs pHEMTs, with a full coplanar waveguide (CPW) technology designed specifically for use in the SKA and fabricated using conventional optical lithography.
\end{abstract}

\section{Introduction}

The Square Kilometre Array (SKA) is the next generation of radio telescope and will have 100 times more sensitivity than any of the largest telescope to date (Dewdney et al. 2009). For the low frequency spectrum of the SKA, cost-effective Ultra Low-Noise Amplifiers are required at frequencies spanning the range of $300 \mathrm{MHz}$ to $2 \mathrm{GHz}$ (Hall 2004). InP-based HEMTs have previously demonstrated superior noise performances at millimetre-wave frequencies using short-gate length and are indeed commonly used in high performance LNAs, albeit at cryogenic temperatures. However, in the low frequency range, matching the LNA for wide band, low noise performance becomes a critical issue as it requires adding large passives into the design, which can act as sources of added noise if integrated in the LNA. This can be circumvented by using off-chip components (Belostotski \& Haslett 2007), but at the expense of both cost and complexity. Thanks to a novel high-breakdown, low-leakage InGaAs/InAlAs pHEMT structures, developed at Manchester (Bouloukou et al. 2006), and which enables the use of large periphery gate transistors, both low NFmin and low $\mathrm{Rn}$, which are key point for a better impedance matching across the ultra-wide band, can be simultaneously obtained. In addition for low frequency applications, the requirement for extremely high cut-off frequencies is relaxed and larger gate lengths $(\sim 1 \mu \mathrm{m})$, as opposed to sub-micron, are perfectly adequate in terms of noise performance and stability of the LNA.

\section{RESULTS AND DISCUSSIONS}

Precise material engineering of the epilayers and doping profile results in on-state leakage $\mathrm{Ig}<0.09 \mathrm{~A} / \mathrm{cm}^{2}$ (Figure 1). In order to verify the foundry process, a single stage LNA, based on a $1 \times 800 \mu \mathrm{m}^{2}$ periphery gate transistor has been designed and measured across the whole frequency band of 0.3 to $3 \mathrm{GHz}$. The design and fabrication of an LNA was based on mea-

\footnotetext{
^ sanae.boulay@manchester.ac.uk
}

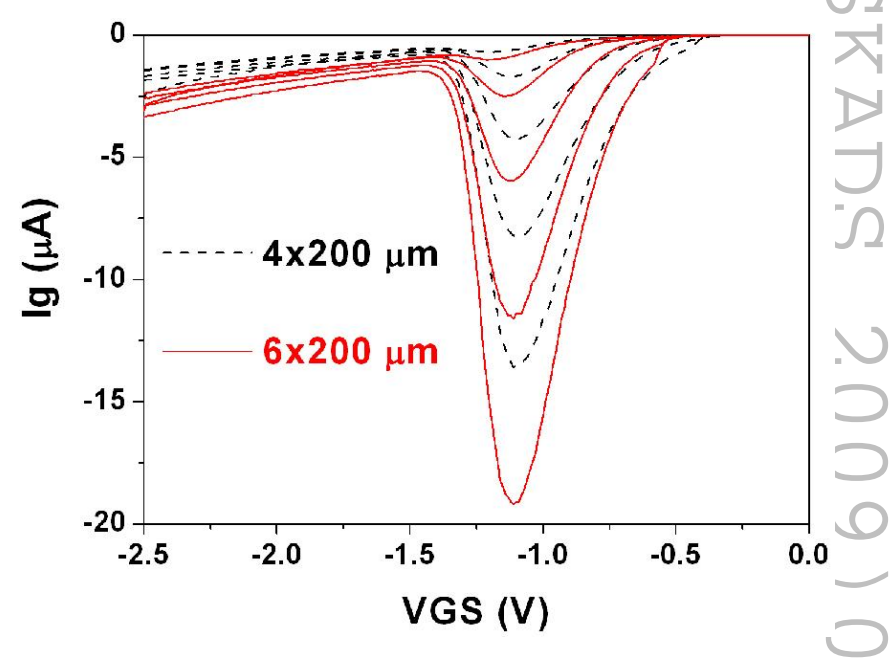

Fig. 1: Typical InGaAs/InAlAs pHEMTs experimental gate current curves for two large gate periphery transistors as a function of drain and gate voltages

surements and modelling of all single elements in the circuit, namely, pHEMTs, inductors, capacitors and resistors.

LNA Designs (2nd Generation) New LNA designs, based on the latest InGaAs/InAlAs epitaxial structures have been chosen for MMIC fabrication. These had more emphasis on low noise figure, input and output reflection coefficients across the ultra-wide frequency band $(0.2$ to $2 \mathrm{GHz})$.

Compared to the 1st generation LNA, this generation of LNAs consisted of new layouts designed to accommodate a range of probe configuration. The LNA 2nd Generation also had designs based on $4 \times 200 \mu \mathrm{m}$ pHEMTs using the $1 \mu \mathrm{m}$ gate InGaAs/InAlAs/InP technology, but presented major improvements compared to the 1 st generation:

- New resistors designed using $50 \Omega / \square$. This leads to more accurate values across the sample and more stable fabrication process. 


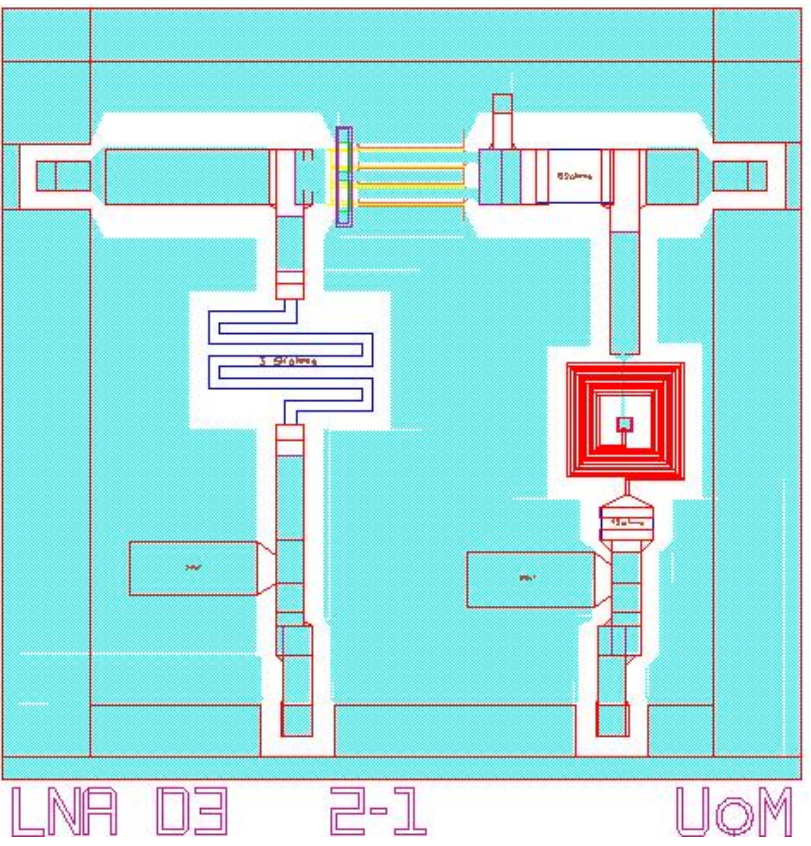

Fig. 2: Schematic picture of MMIC single-stage. The fabricated LNA was $1.52 \times 1.46 \mathrm{~mm} 2$

- Each LNA had a unique label identification based on design number, column-row position on the die which made probing easier.

- The interconnects consisted of a double-metal layer which reduced the resistance variation in the connection.

- The RF pads were designed for RF probes with pitches varying from $100 \mu \mathrm{m}$ to $300 \mu \mathrm{m}$.

- The ground metal plane was extended all around the MMIC. This would minimise the chances of generating oscillations.

- The DC feeds were modified accordingly to our probe pin configuration.

- New process control monitors and RF de-embedding structures were carefully designed and placed on 7 locations on the die.

MMIC Single-Stage LNA A full MMIC single-stage LNA was designed using on-chip integrated input and output matching, as shown in Figure 2. This LNA was successfully measured at MC2 for both RF and noise. The DC was supplied by multi-DC probes, including decoupling capacitors, and designed accordingly with our layout specifications.

These completed first trials MMIC, which have power gain of 15 to $9 \mathrm{~dB}$ across the band (Figure 3) have noise figures of $1.5 \mathrm{~dB}$, larger by some $0.5 \mathrm{~dB}$ than expected by simulation (Figure 4). The reasons for the discrepancies are being addressed in a new run where improvements have been brought to the next generation design with better grounding. Also double stage LNA designs have been implemented which should reduce the broad band noise to $0.5 \mathrm{~dB}$. A likely reason for the increased noise compared with simulated ones can also be attributed to the fact that during full MMIC fabrication, the temperature depositions of the Silicon Nitride capacitors might

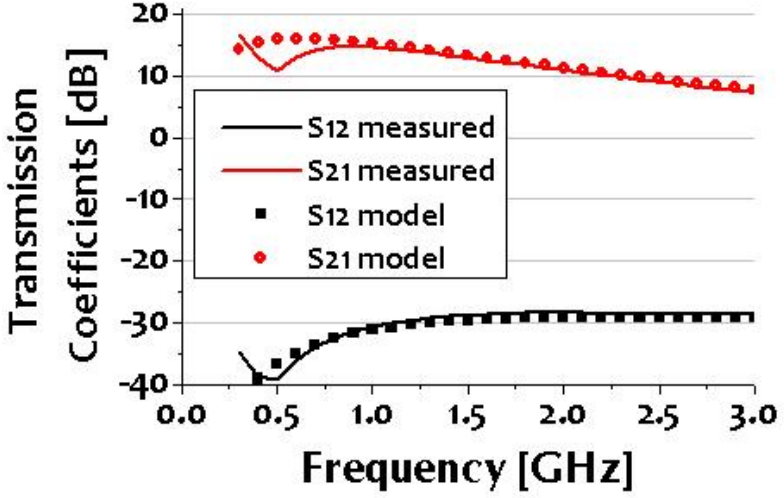

Fig. 3: Measured and modelled S-parameters for a single stage LNA based on a $1 \mu \mathrm{m}$ gate length $4 \times 200 \mu \mathrm{m} \mathrm{InGaAs/InAlAs} \mathrm{transistor}$

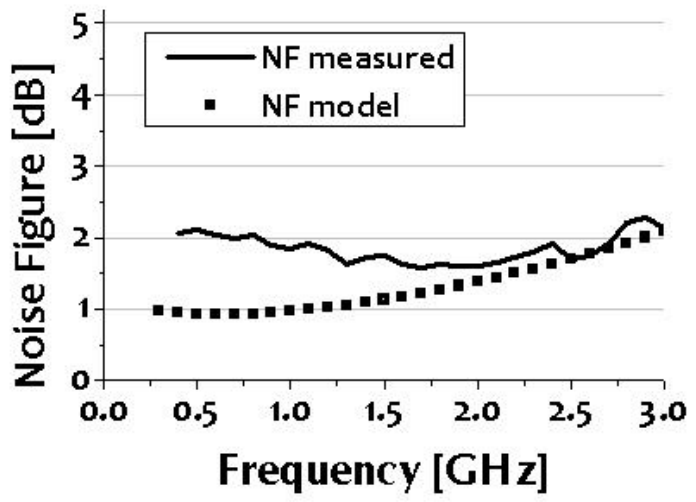

Fig. 4: Noise measurements at $25 \mathrm{~mA}$ drain current on a single stage LNA based on a $1 \mu \mathrm{m}$ gate length $4 \times 200 \mu \mathrm{m} \mathrm{InGaAs/InAlAs} \mathrm{transistor}$

have been too high which caused the transistors to have leak ages much higher than those shown in figure by a factor of 5 which will certainly contribute to the noise figures observed.

The key features of these stable single stage amplifiers are the very low power dissipation of $75 \mathrm{~mW}$, well within the power requirements of SKA. The purpose of this work was also to demonstrate that $\mathrm{CPW}$ can be used at these frequencies for low noise designs and yet still meet size limitations necessary for low cost production. The attractive features of this process include reduced back side processing, no vias, and suitability for flip chip mounting.

MMIC Double Stage LNA Design With Off-Chip Components A double-stage LNA design was developed using off-chip components for reducing parasitic losses at the input path. The design was simulated in ADS using the nonlinear models of the fabricated pHEMT and the passive components. The input matching was achieved using off-chip inductors in order to preserve the LNA noise figure from being affected by the large series resistance (about 20-30 $\Omega$ ) usually found in integrated inductors. The two-stage LNA operating from a $3 \mathrm{~V}$ power supply was designed to include the on-chip 
output matching network. It consists of two common source amplifiers. The first stage was designed to provide a simultaneous noise and impedance match by carefully adjusting the two off-chip inductors at the input using a $4200 \mu \mathrm{m}$ gate width pHEMT as a building block. Simulation results for this LNA design are shown in Figure 5.
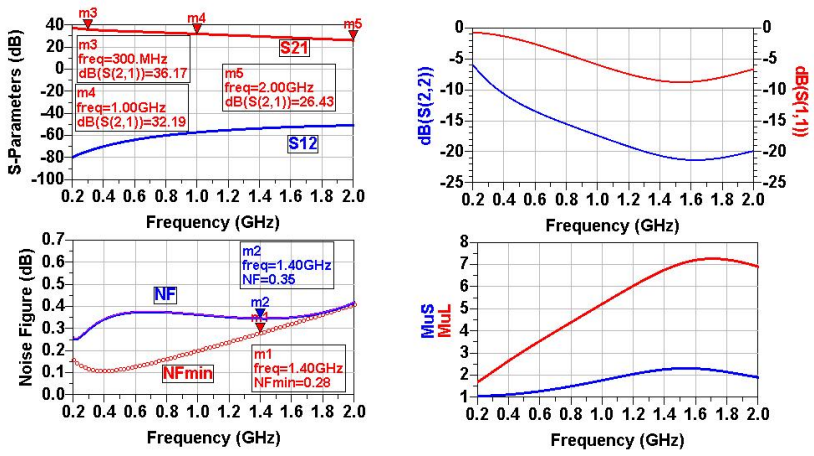

Fig. 5: Simulation results of double-stage MMIC LNA design with off-chip components

The noise figure remains below $0.45 \mathrm{~dB}$ between 0.3 and $2 \mathrm{GHz}$, while the gain is above $26 \mathrm{~dB}$ below $1.4 \mathrm{GHz}$. The input and output return losses S11 and S22 were below $-9 \mathrm{~dB}$ and $-14 \mathrm{~dB}$, respectively at $1.4 \mathrm{GHz}$. The third order intercept obtained from LNA simulations was $14 \mathrm{dBm}$. The circuit also showed unconditional stability up to $30 \mathrm{GHz}$ with a total power dissipation of about $100 \mathrm{~mW}$. Table 1 conveys the main simulations results compared with those reported in the literature at this frequency band. The LNA presented in this paper shows noise characteristics amongst the best published to date especially when considered in the light of the relatively large, optically defined gate length of the transistor used here $(1 \mu \mathrm{m})$. The high gain LNA presented in (Rosenbaum et al. 1993) was obtained using a three-stage circuit which would dissipate large amounts of power but would probably have higher IP3.

Clearly, this design would be a very strong contender for the low frequency band of the SKA. This LNA has successfully been fabricated and is being measured now.

Fully Integrated Double-Stage LNA Design Another full MMIC double-stage LNA was prepared with on-chip components for input and output matching. As expected, the gain was higher than $30 \mathrm{~dB}$ across the band, while dissipating a total power of about $110 \mathrm{~mW}$. The overall noise figure was expected to be lower than $0.7 \mathrm{~dB}$ between 0.2 to $2 \mathrm{GHz}$, and less than $0.5 \mathrm{~dB}$ across most of the frequency band, as shown in Figure 6 .

Double-Stage Differential to Single-Ended MMIC LNA Design Considering the interfacing requirements of the LNA in the receiver chain of SKA, a differential to single-ended MMIC LNA design has been prepared. This design comprises two stages where the first stage is fully differential, optimised for noise performance with reasonable gain, while the second stage is a gain and stability boosting stage with differential input and
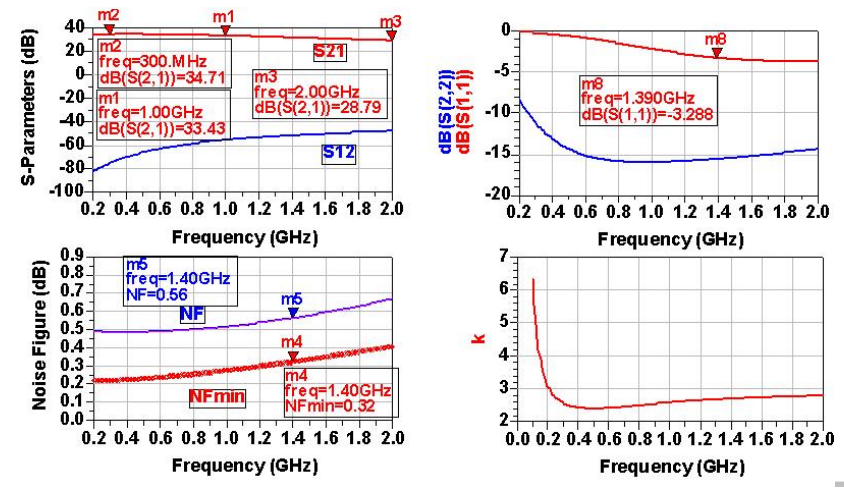

Fig. 6: Simulation results for double-stage MMIC LNA design

single-ended output interfaces. The noise performance of the first fully differential stage is considerably better as compared to the second differential to single-ended conversion stage due to the balanced topology. Due to the considerable high gain of the first stage (12-15 dB), the relatively degraded noise performance of the second stage has minimal effect on the amplifier's noise characteristics, as expressed by the Friis' expression for noise figure of cascaded microwave networks (Friis 1944). The designed LNA optimised for noise figure and power consumption with reasonably high gain of $302 \mathrm{~dB}$ for the low frequency band of SKA (0.3-1 GHz). A noise figure of less than $0.6 \mathrm{~dB}$ with unconditional stability for the entire frequency band of interest is predicted for this design with power dissipation of $300 \mathrm{~mW}$. The simulated results presented in Figure 7 show high losses at lower frequencies for the input because of the absence of input matching network. Reasonable output SWR S22 and good reverse isolation $\mathrm{S} 12$ are predicted for the design. Layouts and masks are currently being prepared for this design.
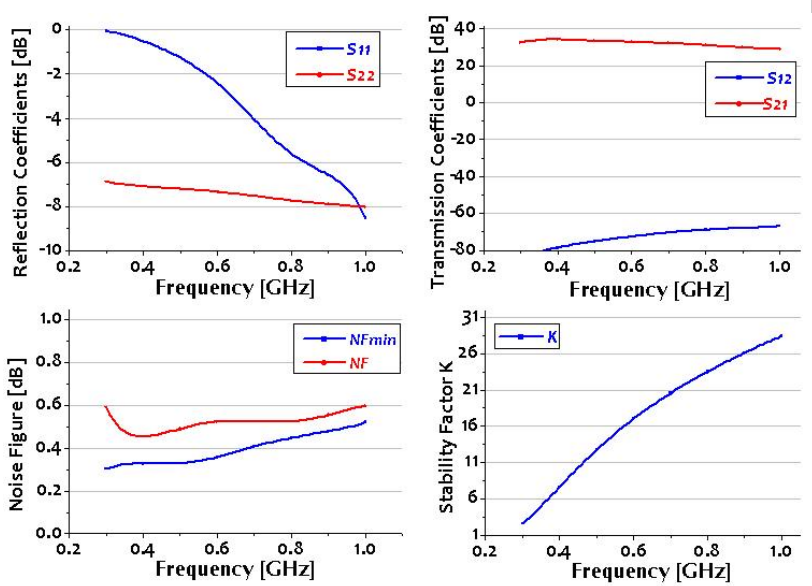

Fig. 7: Simulation results for double stage differential input singleended output MMIC LNA design 
Table 1: Summary of the main ultra low noise amplifiers below $2.5 \mathrm{GHz}$, at room temperature

\begin{tabular}{|c|c|c|c|c|c|c|}
\hline $\begin{array}{c}\text { Bandwidth } \\
(\mathrm{GHz})\end{array}$ & $\begin{array}{c}\mathrm{NF} \\
(\mathrm{dB})\end{array}$ & Technology & $\begin{array}{c}\text { Power } \\
(\mathrm{mW})\end{array}$ & $\begin{array}{c}\text { IP3 } \\
(\mathrm{dBm})\end{array}$ & $\begin{array}{c}\text { S21 } \\
(\mathrm{dB})\end{array}$ & Ref. \\
\hline $0.7-1.4$ & 0.35 & $90 \mathrm{~nm}$-CMOS & 45 & 7.5 & $20.5-16.3$ & Belostotski \& Haslett (2007) \\
\hline $0.6-1.6$ & 0.5 & $0.5 \mu \mathrm{m}$ GaAs pHEMT & 852 & 15.4 & $29.3-20.9$ & Xu et al. (2005) \\
\hline $2.25-2.5$ & 0.5 & $0.15 \mu \mathrm{m}$ InGaAs/InAlAs HEMT & $?$ & $?$ & $>35$ & Rosenbaum et al. (1993) \\
\hline $0.3-2$ & 0.45 & $1 \mu \mathrm{m}$ InGaAs/InAlAs pHEMT & 110 & 14 & 26 & This work \\
\hline
\end{tabular}

\section{Conclusion}

LNA designs based on a newly developed high breakdown, large gate periphery (and hence low noise resistance) transistors have been implemented and successfully fabricated in the 2nd generation of LNAs. These were based on work carried out on accurate modelling of passives and pHEMTs using the $1 \mu \mathrm{m}$ gate InP technology developed at the University of Manchester. Major improvements have been made on these LNAs which mainly concentrated on achieving low noise figure across the ultra wide frequency band $(0.2$ to $2 \mathrm{GHz})$. The best LNA is expected to exhibit less than $0.4 \mathrm{~dB}$ noise figure at room temperature across the whole band, still using a cost-effective $1 \mu \mathrm{m}$ gate technology. This shows great promises for future development using sub-micron gate lengths.

\section{References}

L. Belostotski, J.W. Haslett, IEEE Radio and Wireless Symposium, Long Beach, pp. 221- 224, January, 2007.

A. Bouloukou et al. the 7th MINT Millimetre-Wave International Symposium - MINT-MIS 2006, Finland.

Dewdney, P.E., Hall, P.J., Schilizzi, R.T., Lazio, T.J.W., "The Square Kilometre Array," Proceedings of the IEEE, Vol. 97, No. 8, Aug. 2009 pp. 1482-1496, ISSN: 0018-9219

Friis, H.T., Noise Figure of Radio Receivers. Proceedings of the IRE, 1944. 32(7): p. 419-422.

P.J. Hall, Experimental Astronomy, Vol. 17, no. 1-3, pp. 5-16, June 2004.

S. E. Rosenbaum. L. M. Jelloian. L. E. Larson. U. K. Mishra. D. A. Pierson, M. S Thompson, T. Liu, A. S. Brown, IEEE Microwave Guided Wave Lett. 3(8), 265, 1993.

J. Xu, B. Woestenburg, J. Geralt bij de Vaate, W.A. Serdijn, Electr. Lett. 41(14), 2005. 